\title{
Multi-omics analysis identifies FoxO1 as a regulator of macrophage function through metabolic reprogramming
}

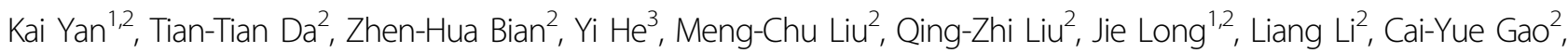 \\ Shu-Han Yang ${ }^{2}$, Zhi-Bin Zhao ${ }^{2}$ and Zhe-Xiong Lian ${ }^{2}$
}

\begin{abstract}
Macrophages are plastic cells that can switch among different states according to bioenergetic or biosynthetic requirements. Our previous work demonstrated that the transcription factor Forkhead Box Protein 1 (FoxO1) plays a pivotal role in regulating the function of macrophages, but the underlying mechanisms are still unclear. Here we identify FoxO1 as a regulator of macrophage function through metabolic reprogramming. Transcriptomic and proteomic analyses showed that the deficiency of FoxO1 results in an alternatively activated (M2) phenotype of macrophages, with lower expression of inflammatory response- and migration-associated genes. Using the high content screening and analysis technology, we found that deletion of FoxO1 in macrophages slows their migration rate and impairs their function to limit tumor cell growth in vitro. Next, we demonstrated that glycolysis is inhibited in FoxO1-deficient macrophages, which leads to the observed functional changes and the reduced tumor suppression capability. This prospective study shows that FoxO1 serves as a bridge between metabolism and macrophage function.
\end{abstract}

\section{Introduction}

Macrophages form a heterogeneous population of antigen-presenting and tissue-resident phagocytic cells. They develop from the terminal differentiation of circulating monocytes or from E8.0 yolk $\mathrm{sac}^{1-3}$. Macrophages are central effector cells of the host defense mechanism; they phagocytize and digest impurities, dead cells, and pathogens, and they also play important roles in the orchestration of innate and adaptive immune responses ${ }^{4,5}$. Resting state (M0) macrophages, derived from the bone marrow, usually function as precursors of polarized macrophages. Upon stimulation with lipopolysaccharide (LPS)

\footnotetext{
Correspondence: Zhi-Bin Zhao (zhaozb@scut.edu.cn) or

Zhe-Xiong Lian (zxlian@scut.edu.cn)

'Liver Immunology Laboratory, School of Life Sciences, University of Science and Technology of China, Hefei 230022 Anhui, China

${ }^{2}$ Chronic Disease Laboratory, Institutes for Life Sciences and School of Medicine, South China University of Technology, Guangzhou 510006

Guangzhou, China

Full list of author information is available at the end of the article

Edited by C. Munoz-Pinedo
}

and Th1 pro-inflammatory cytokines, M0 cells can be polarized to classically activated (type 1 or M1) proinflammatory macrophages and interleukin (IL)-4/IL-13, or $I L-10 /$ transforming growth factor- $\beta$ stimulation results in alternatively activated (type 2 or M2) anti-inflammatory macrophages $^{6-8}$. M1 macrophages initiate and sustain the inflammatory responses, by secreting cytokines such as tumor necrosis factor- $\alpha(T N F-\alpha), I L-6$, and interferon- $\gamma$ $(I F N-\gamma)$, and recruiting other immune cells to the inflamed tissue $^{9,10}$. Contrary to M1 macrophages, M2 macrophages exhibit an anti-inflammation phenotype; they promote the resolution of inflammation, phagocytize apoptotic cells, and release Th2-inflammatory mediators such as $I L-4^{8,11}$. In tumor, the macrophage polarization become more complex. Tumor-associated macrophages (TAMs) adopt activation signals over a wide time range, to express M1associated genes and more M2-associated genes ${ }^{12-15}$. Tumor cells harness metabolic byproducts to keep immune cells in their favor. The secretion of large amounts of lactate favors the polarization to an immunosuppressive

\section{(c) The Author(s) 2020}

(c) (i) Open Access This article is licensed under a Creative Commons Attribution 4.0 International License, which permits use, sharing, adaptation, distribution and reproduction cc) in any medium or format, as long as you give appropriate credit to the original author(s) and the source, provide a link to the Creative Commons license, and indicate if changes were made. The images or other third party material in this article are included in the article's Creative Commons license, unless indicated otherwise in a credit line to the material. If material is not included in the article's Creative Commons license and your intended use is not permitted by statutory regulation or exceeds the permitted use, you will need to obtain permission directly from the copyright holder. To view a copy of this license, visit http://creativecommons.org/licenses/by/4.0/. 
phenotype $^{16,17}$. It is known that macrophages display diverse metabolic states, especially macrophages polarized in vitro. In M1 macrophages, an increased glycolysis rate is closely associated with an inflammatory phenotype, including the secretion of pro-inflammatory cytokines and opsonin-mediated phagocytosis ${ }^{10,18,19}$. In contrast, M2 macrophages employ oxidative phosphorylation as their main energy source ${ }^{20-22}$. TAMs show high glycolytic activity and high oxidative phosphorylation, which means that TAMs present M1 and M2 characteristics with heterogenous subpopulations ${ }^{17}$. It is known for dramatic switches in metabolic signature of macrophages governing its phenotype and function ${ }^{16,23-26}$. However, little is known about the transcriptional mechanisms underlying metabolic reprogramming in macrophages.

The transcription factor Forkhead Box Protein 1 (FoxO1) is a member of the Forkhead Box family and plays significant roles in a series of cellular processes, including inflammation, metabolism, and signal transduction $^{27-30}$. In tumor cells, FoxO1 is generally considered as a tumor suppressor and upregulation of FoxO1 is beneficial in several types of cancer ${ }^{31-33}$. In macrophages, FoxO1 seems to have various functions. FoxO1 dampens macrophage inflammation without promoting apoptosis in the context of endoplasmic reticulum stress ${ }^{4}$ and it also promotes goblet cell hyperplasia in asthma by regulating M2-like macrophage activation ${ }^{34}$. Besides FoxO1 also interacts with the Toll-like receptor 4-mediated inflammatory response ${ }^{35}$ and regulates $I L-10$ secretion during classic activation in hyperglycemia ${ }^{36}$. In previous studies, we found that FoxO1 regulates macrophage polarization in infection and promotes the anti-tumor function of TAMs by upregulating major histocompatibility complex II (MHC-II) expression ${ }^{37,38}$. In this study, we focused on the transcriptional mechanism of macrophage metabolic reprogramming.

\section{Results \\ FoxO $1^{\text {mKo }}$ macrophages are skewed toward an $\mathrm{M} 2$ phenotype in physiological state}

FoxO1 is a diversified transcriptional factor but little is known about its role in macrophage metabolism and function. First, we cultured bone marrow-derived macrophages (BMDMs) from FoxO1 myeloid-specific knockout $(\mathrm{KO})$ mice $\left(F o x O 1^{\mathrm{mKO}}\right)$ and their littermate controls $\left(F o x O 1^{\mathrm{f} / \mathrm{fl}}\right)$. We found that foxo1 deficiency did not affect macrophage development in vivo (Supplementary Fig. S2). Next, RNA and protein were extracted separately for further transcriptome and proteome analyses. A total of 21,460 efficient genes in all samples $\left(F o x O 1^{\mathrm{mKO}} \mathrm{M} 0\right.$,

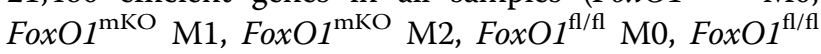
$\mathrm{M} 1$, and $F o x O 1^{\mathrm{fl} / \mathrm{fl}} \mathrm{M} 2$ BMDMs) were analyzed (Gene Expression Omnibus (GEO): GSE97260). Principal component analysis (PCA) and Pearson's correlation results of transcriptome data indicate that the physiological state of $F o x O 1^{\mathrm{mKO}}$ macrophages is more similar to that of control M2 cells than control M0 cells (Fig. 1a, b). Our proteomics data are consistent with the transcriptome results. Here we examined the quantified proteins of each samples, which identified $\sim 2000$ proteins, and there was no difference in protein abundance between $F o x O 1^{\mathrm{mKO}}$ and $F o x O 1^{\mathrm{fl} / \mathrm{fl}}$ macrophages (Fig. 1c). PCA and Pearson's correlation results of proteomics data also showed that the physiological state of $F_{0 x O 1}{ }^{\mathrm{mKO}}$ macrophages resembles that of a M2 phenotype (Fig. $1 \mathrm{~d}-\mathrm{f})$. These results suggest that FoxO1 deficiency results in an M2 phenotype of macrophages.

\section{FoxO1 regulates the polarization and function of macrophages}

To further confirm the role of FoxO1 in macrophages, we used multi-profiling to explore the phenotype and function of FoxO1-deficient macrophages. First, we compared the typical gene expression sets in physiological states of FoxO1-deficient macrophages with those of control macrophages. As for pro-inflammatory genes, the "Hallmark Inflammatory Response" (normalized enrichment score $(\mathrm{NES})=1.182, P=0.059$; Fig. 2a), "Hallmark Interferon Gamma Response" (NES $=1.424, P=0.001$; Fig. 2b), and "Hallmark Interferon Alpha Response" (Supplementary Fig. S1A) gene sets showed significant downregulation in FoxO1-deficient macrophages compared with control macrophages. Regarding anti-inflammatory gene sets, "GO IL4 Production" (NES $=-1.243, P=0.169$; Fig. $2 \mathrm{c}$ ) and "GO IL13 Production" (NES $=-1.333, P=0.081$; Fig. $2 \mathrm{~d}$ ) showed an enriched trend in FoxO1-deficient macrophages. Furthermore, gene set enrichment analysis (GSEA) plot of the "Positive Regulation of Macrophage Phagocytosis" $(\mathrm{NES}=1.698, P<0.001$; Fig. 2e) and "GO Macrophage Activation Involved in Immune Response" (NES $=1.726$, $P=0.003$; Supplementary Fig. S1B) gene sets showed significant downregulation in FoxO1-deficient macrophages. In addition, the macrophage chemotaxis- and migrationrelated gene sets "GO Regulation of Macrophage Chemotaxis" (NES $=1.497, P=0.032$; Supplementary Fig. S1C) and "GO Regulation of Lymphocyte Migration" (NES = 1.850, $P<0.001$; Fig. 2f) showed significant downregulation in FoxO1-deficient macrophages. Finally, quantitative PCR and flow cytometry were used to confirm the mRNA and protein expression levels of M1/M2-associated markers in shFoxO1 lentivirus-infected macrophages and FoxO1 ${ }^{\mathrm{mKO}}$ macrophages. As shown in Fig. 2g, IL-10 and Arg-1 (M2 markers) expression significantly increased in FoxO1deficient macrophages, but there was no difference on TNF$\alpha$ (an M1 marker). The flow cytometry results showed that CD206 (an M2 marker) was upregulated but MHC-II (an M1 marker) was downregulated in FoxO1 ${ }^{\mathrm{mKO}}$ macrophages 


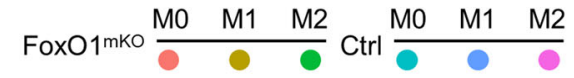

(A)

(C)

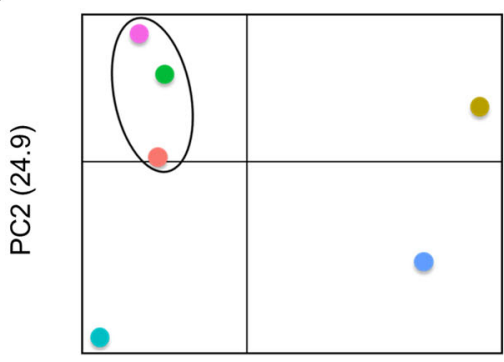

PC1 $(38.8 \%)$

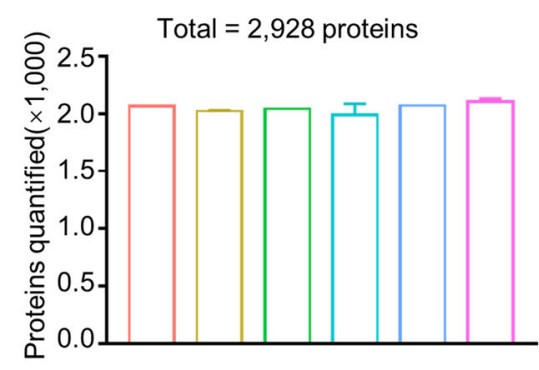

(E)

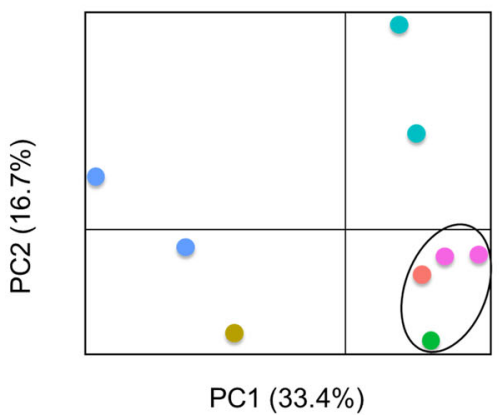

(B)

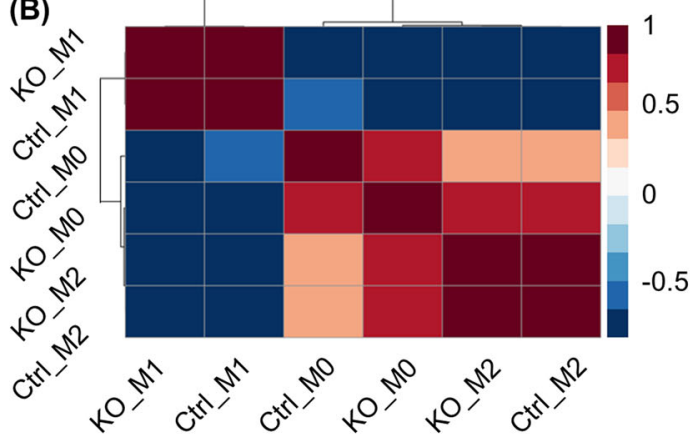

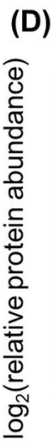

(F)
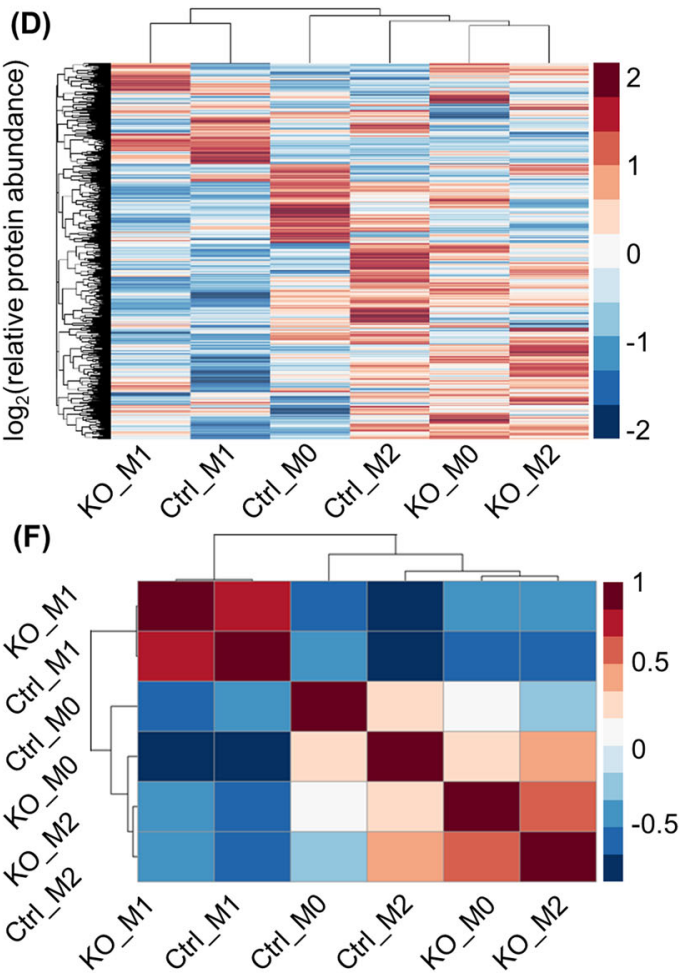

Fig. 1 Transcriptome and proteome analyses imply propinquity of the $\mathrm{FoxO} 1^{\mathrm{mKO}}$ macrophage phenotype to that of alternatively activated (M2) macrophages. The transcriptome and proteome of $\mathrm{MO}, \mathrm{M1}$, and $\mathrm{M} 2$ macrophages from FoxO ${ }^{\mathrm{mKO}}$ and littermate control mouse bone marrowderived macrophages (BMDMs) were analyzed by RNA sequencing and proteomics analysis. a A 2D principal component analysis (PCA) plot of the transcriptome data that characterizes the trends exhibited by the expression profile of 21,460 genes of FoxO I $^{\mathrm{mKO}} \mathrm{MO}$ (orange), FoxO l $^{\mathrm{mKO}} \mathrm{M} 1$ (gray), FoxO $1^{\mathrm{mKO}} \mathrm{M} 2$ (green), Ctrl MO (cyan), Ctrl M1 (blue), and Ctrl M2 (pink) BMDMs. Every transcriptome sequencing sample is a mixture of two FoxO $1^{\mathrm{mkO}}$ mice or two littermates. $\mathbf{b}$ Pearson's correlation computation of transcriptome data in all samples. Colors indicate the coefficient values between classes of cells. c Numbers of unique proteins quantified by Sequest-HT in BMDMs; the total number of identified proteins was 2928 . The bar color is the same as the color of Fig. 1a's dot. $\mathbf{d}$ Consensus-clustering analysis of proteomic profiling identifies six BMDM proteomic subtypes $(n=9)$. The heat map depicts the relative abundance of signature proteins ( $\log _{2}$-transformed). The PCA plot (e) and Pearson's correlation analysis (f) of the proteomics data by the total 2400 efficient protein expression profiles of BMDM cells. Figure 1a, e share the same dot color.

(Supplementary Fig. S1E). Besides, the expression levels of $I L-4$, a cytokine enriched in M2 macrophages, were significantly increased in FoxO1-deficient macrophages compared with normal macrophages, but no differences in the expression of IL-6 (an M1 cytokine) were observed (Supplementary Fig. S1D).

\section{Fox01-deficient macrophages exhibit an M2-like phenotype}

Our transcriptome and proteome analyses showed that FoxO1-deficient macrophages resemble M2 macrophages in phenotype and function. Next, we further explored the role of FoxO1 in macrophage migration and phagocytosis. 


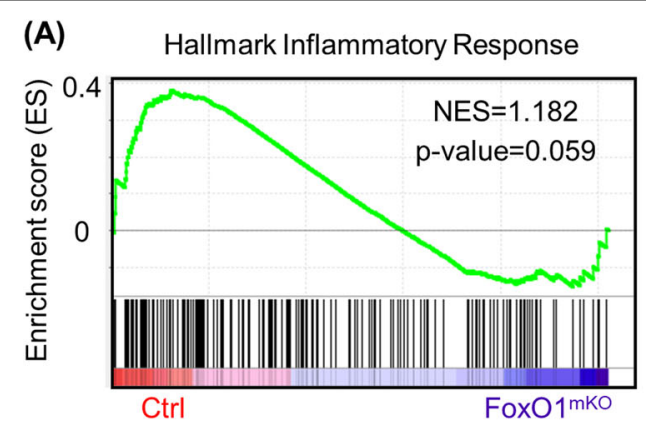

(B) Hallmark Interferon Gama Response
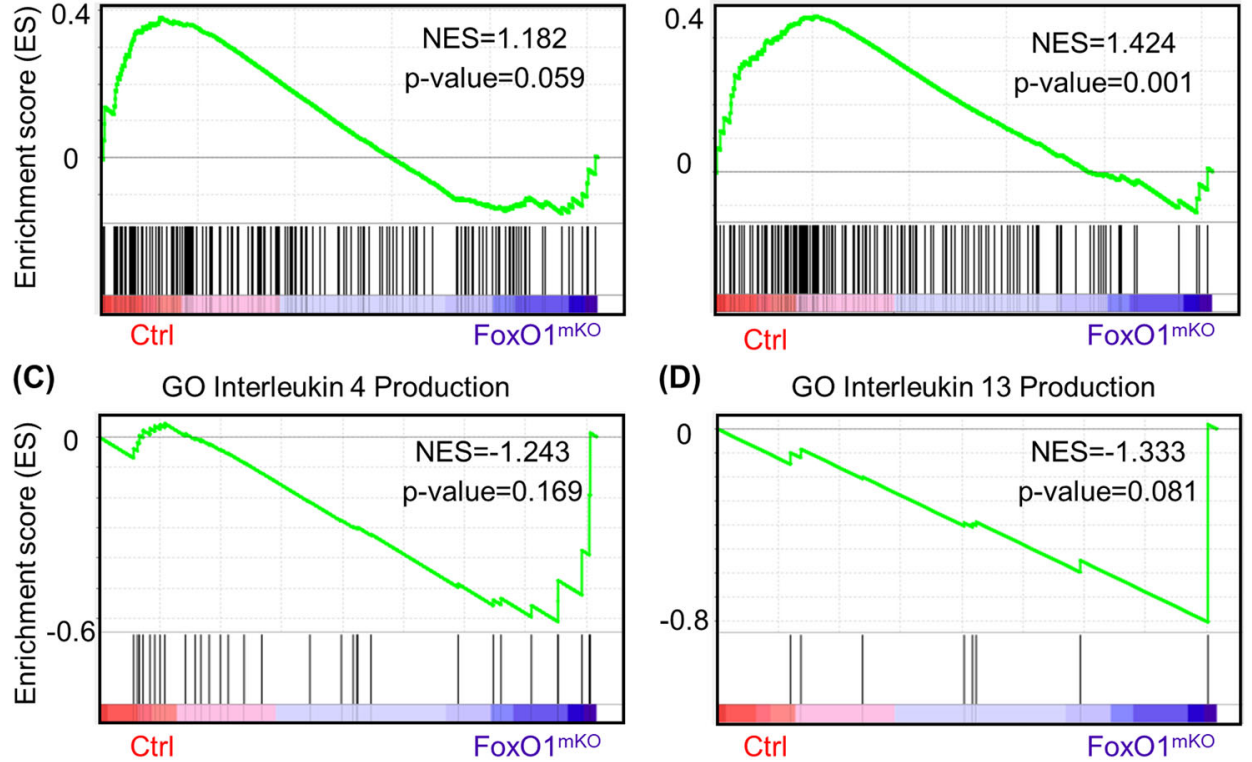

(D)

GO Interleukin 13 Production

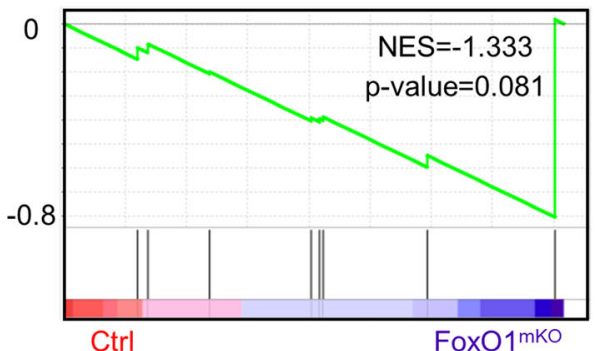

(E) GO Positive Regulation of Phagocytosis

(F) Go Regulation of Lymphocyte Migration
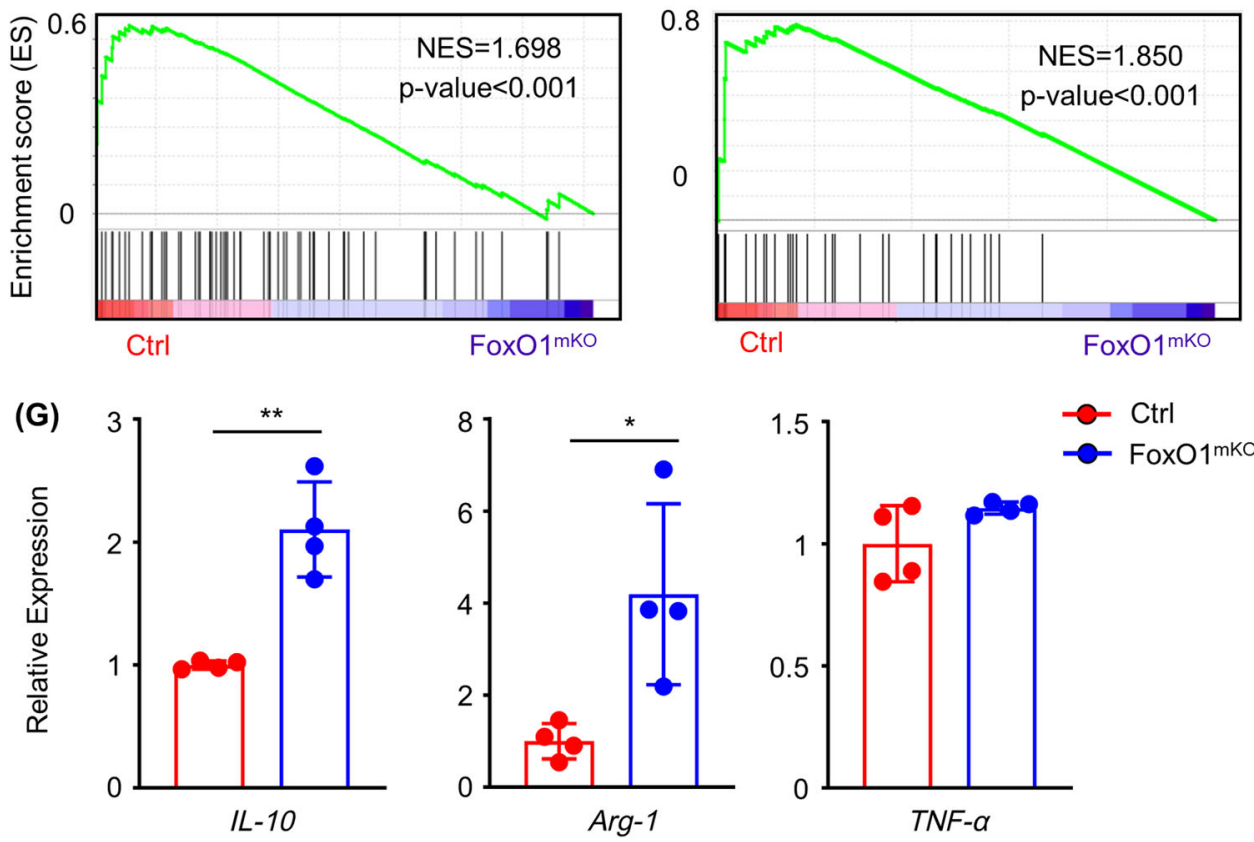

Fig. 2 Multi-profiling implies FoxO1 deficiency results in resemblance of the phenotype and function of macrophages to those of alternatively activated (M2) macrophages. The transcriptome profiles of macrophages from FoxO1 conditional knockout mice and littermates were analyzed by GSEA and confirmed with real-time-PCR. The list of differentially expressed genes (upregulated or downregulated) in Control and FoxO1-knockout macrophages was obtained from the MSigDB database. Two FoxO $1^{\text {mKO }}$ mice or two littermates were mixed for transcriptomics. a-f GSEA plots of $(\mathbf{a}, \mathbf{b})$ pro-inflammatory gene sets, (c, $\mathbf{d})$ anti-inflammatory cytokine production gene sets, (e) macrophage phagocytosis-related gene sets, and (f) macrophage migration-related gene sets. NES, normalized enrichment score. $\mathbf{g}$ Bar graphs showing the relative expression of $/ L-10$, Arg-1, and TNF- $a$ in control and FoxO1 ${ }^{\mathrm{mKO}}$ mice bone marrow-derived macrophages. Red bar represents control macrophages and the blue bar represents FoxO1-deficient macrophages. Four mice were used for the induction of bone marrow-derived macrophages in each experiment. ${ }^{*} P<$ $0.05 ;{ }^{*} P<0.01 ;{ }^{* *} P<0.001$. 


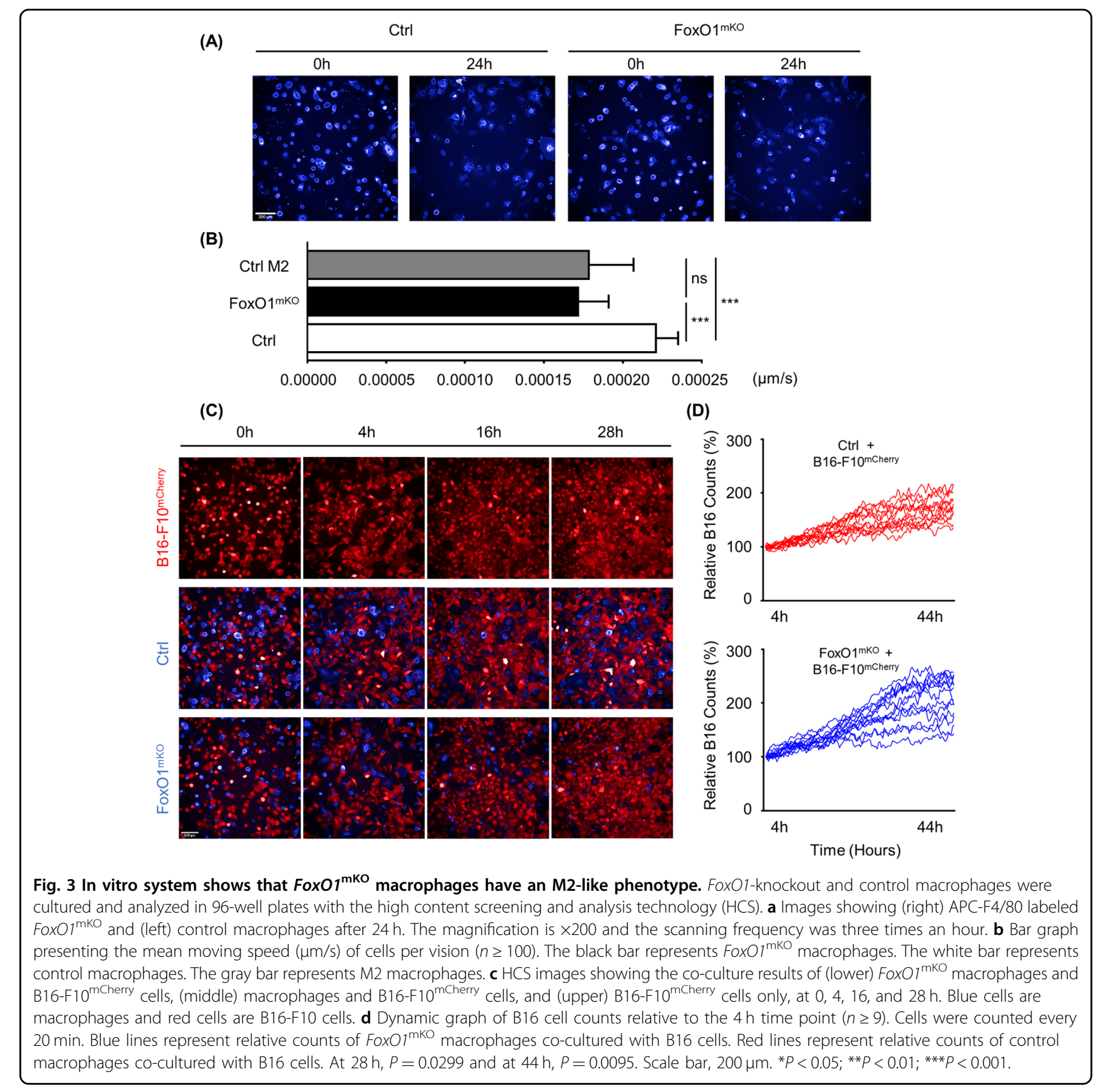

First, we used the high content screening and analysis technology (HCS) system to calculate the moving speeds of $F_{0 x O 1^{\mathrm{mKO}}}$ and $\mathrm{FoxO}^{\mathrm{fl} / \mathrm{fl}}$ macrophages. We found that the lack of FoxO1 in macrophages leads to decreased moving speeds (Fig. 3a, b). In addition, when co-cultured with B16F10 ${ }^{\text {mCherry }}$ cells, the moving speed of FoxO $1^{\mathrm{mKO}}$ macrophages increased, but they remained slower than normal macrophages (Supplementary Fig. S3A). Moreover, we observed the phagocytosis function of macrophages by HCS co-culturing at different time points and compared the relative B16-F10 ${ }^{\text {mCherry }}$ counts (Fig. 3c, d, Supplementary Fig. S3D, and Supplementary Video S1A, B). As shown in Supplementary Fig. S3D, the relative B16 counts gradually decreased from $\mathrm{M} 2, \mathrm{M} 0$, to $\mathrm{M} 1$ in co-culture system. The relative proportion of B16 counts significantly enhanced when co-cultured with FoxO1-deficient macrophages compared with control macrophages $(p<0.05)$ (Fig. 3c, d and Supplementary Video S1A, B). The phagocytosis process was also directly visualized by merging HCS and immunofluorescence videos (Supplementary Fig. S3B, C and Supplementary Videos S2 and S3A, B). Consistent with the lower moving speed, the anti-tumor function (including phagocytosis) of $F o x O 1^{\mathrm{mKO}}$ macrophages was impaired compared to that of control macrophages. These 

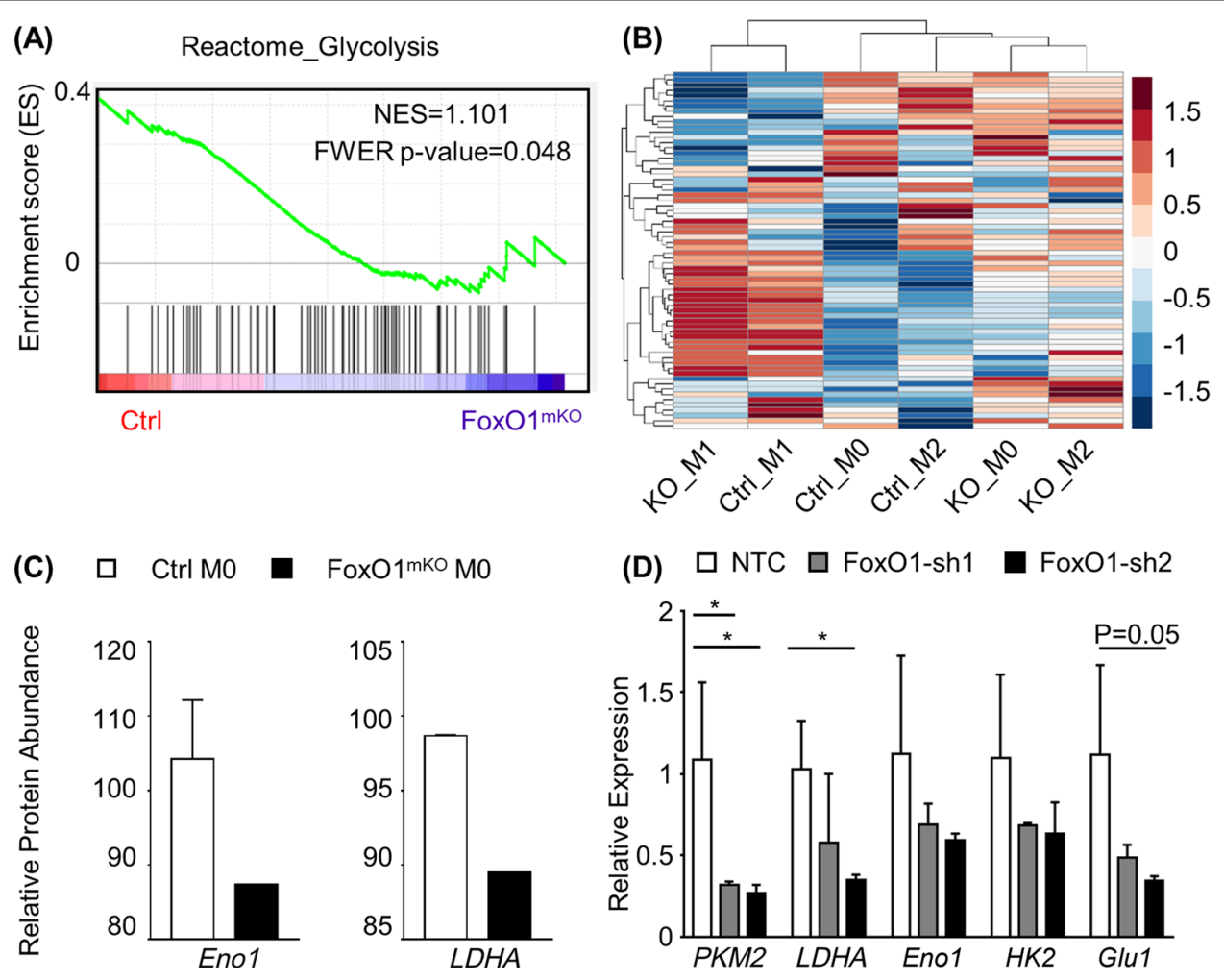

FoxO1mko M0

(D) $\square$ NTC $\square$ FoxO1-sh1

FoxO1-sh2
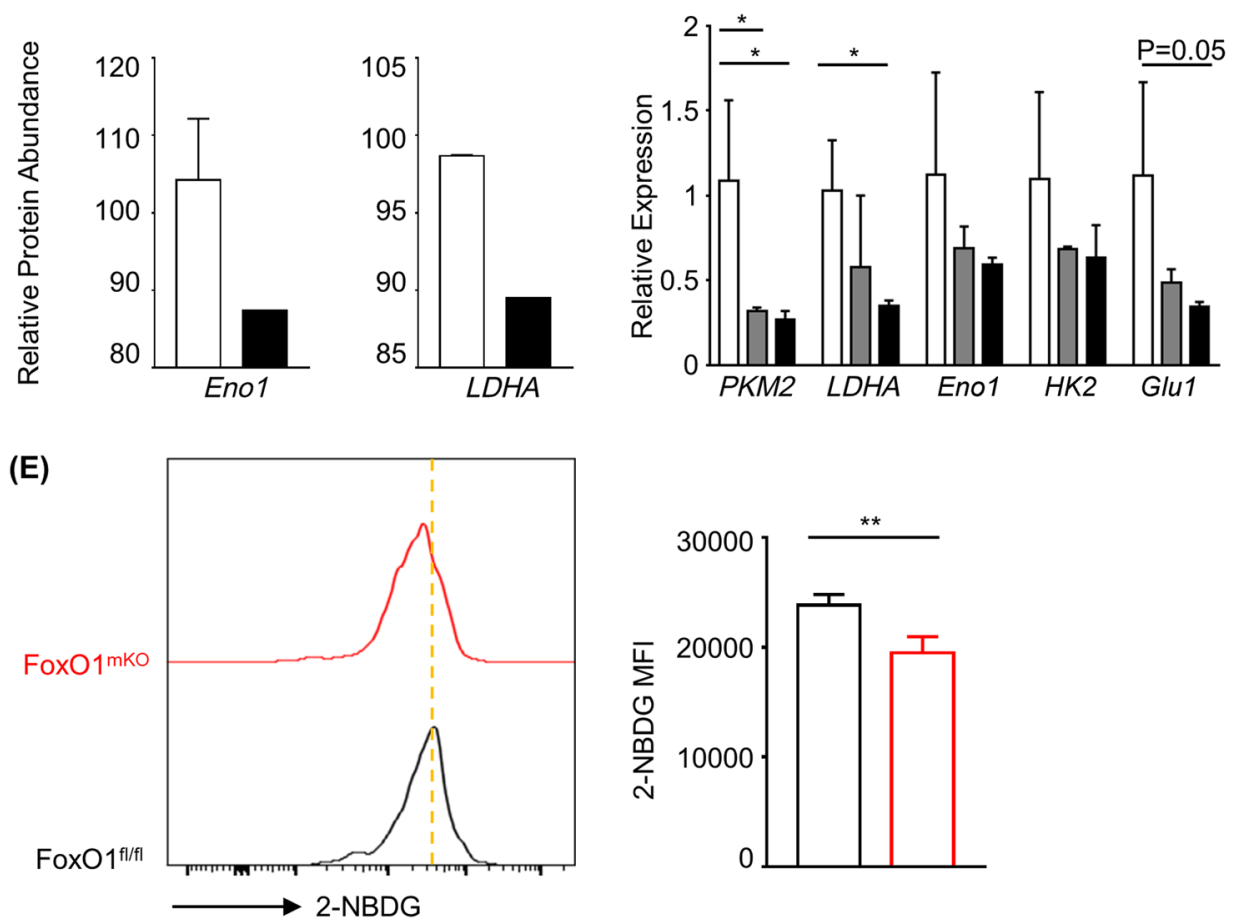

Fig. 4 FoxO1-deficient macrophages exhibit reduced glycolytic activity. a Gene set enrichment analysis (GSEA) of the "Reactome Glycolysis" gene set in FoxO1 conditional knockout macrophages vs. control macrophages was performed on the RNA-seq data set. b Heat map depicting colorcoded expression levels of genes from the "Reactome Glycolysis" gene set in FoxO1 ${ }^{\mathrm{mKO}}$ and control M0, M1, and M2 BMDMs, as obtained by GSEA. c Relative expression levels of key glycolysis genes LDHA and Eno 1 from the proteome data set. The black bar represents FoxO ${ }^{\text {mKO }}$ macrophages. The white bar indicates control macrophages. $\mathbf{d}$ Expression levels of glycolysis-related genes were determined by quantitative real-time PCR $(n \geq 3)$. e 2NBDG fluorescence intensity in FoxO ${ }^{\mathrm{mKO}}$ macrophages (red) and control macrophages (black). MFI, mean fluorescence intensity $(n \geq 3)$. ${ }^{*} P<0.05$; ${ }^{* *} P<0.01 ;{ }^{* *} P<0.001$.

data indicate that FoxO1 not only regulates the differentiation of macrophages but also promotes moving and anti-tumor effect including phagocytosis, especially when co-cultured with cancer cells.

\section{Glycolytic activity is downregulated in FoxO1-deficient macrophages}

It is well known that metabolic homeostasis defines functions, especially glucose metabolism, where the metabolite concentrations (e.g., succinate) directly alter the activity and function of signaling pathways. Interestingly, our GSEA showed that the profile of glycolysisassociated genes in $F_{0 x O 1^{\mathrm{mKO}}}$ macrophages resembled that of M2 macrophages more than it resembled that of FoxOl ${ }^{\mathrm{f} / \mathrm{fl}}$ macrophages (Fig. 4a). Our random clustered heat map of glycolysis-associated genes in $\mathrm{FoxO}^{\mathrm{mKO}}$ macrophages and $\mathrm{FoxOl}^{\mathrm{fl} / \mathrm{fl}}$ macrophages shows that glycolytic activity in FoxO1-deficient macrophages is 
similar to that in M2 macrophages (Fig. 4b). The genes associated with Reactome glycolysis reduced in FoxO $1^{\mathrm{mKO}}$ macrophages compared with those of $F o x O 1^{\mathrm{fl} / \mathrm{fl}}$ macrophages in physiological state according to proteomics data (Fig. 4c). We also performed short hairpin RNA (shRNA) knockdown of FoxO1 to further confirm the role of FoxO1 in regulating glycolytic activity. As shown in Fig. 4d, knockdown of FoxO1 in macrophages with shRNA results in reduced expression of the key glycolytic molecules glucose transporter 1 (Glut1), lactate dehydrogenase A (LDHA), enolase 1 (Eno1), pyruvate kinase isozymes M2 (PKM2), and hexokinase 2 (HK2). FoxO1 ${ }^{\mathrm{f} / \mathrm{fl}}$ macrophages infected with adeno-cre virus further validated the downregulation of Glut1, Eno1, $P K M 2$, and $H K 2$ genes along with FoxO1 deficiency (Supplementary Fig. S4A, B). Besides, the glycolytic gene expression pattern in FoxO1-deficient macrophages showed a similar low-expression manner with M2 macrophages, but different from the high-glycolysis M1 macrophages (Supplementary Fig. S4A, B). We also confirmed the reduced glucose uptake level by flow cytometry in FoxO1-KO BMDMs (in vitro) (Fig. 4e) and peritoneal cavity (PC) macrophages (ex vivo) (Supplementary Fig. S4C). Besides, both FoxO1 conditional KO BMDMs and PC macrophages showed a reduced lactate production trend compared with control macrophages (Supplementary Fig. S4D, E). Collectively, these results demonstrate that glycolytic activity in macrophages is downregulated upon FoxO1 deficiency, indicating that FoxO1 is a regulator of macrophage function through metabolic reprogramming.

\section{FoxO1 regulates glucose metabolism of macrophages}

To further confirm the role of FoxO1 in regulating glucose metabolism in macrophages, we isolated FoxO1 conditional $\mathrm{KO}$ macrophages and detected the metabolic profiles using a Seahorse Glycolysis Stress Test Kit (Agilent). Initially, we measured bioenergetics profiles at the basal state and performed a typical glycolytic function assay $^{39}$ (Fig. 5a). We measured the kinetic extracellular acidification rate (ECAR) response curve in BMDMs from FoxO1-deficient mice and littermate controls, and we found that the ECAR was lower in FoxO1-deficient macrophages than that in normal macrophages (Fig. 5a). In M2 macrophages, no differences were found (Supplementary Fig. S4F). In FoxO1-deficient macrophages, there is a significant decrease in the ECAR of non-glycolytic acidification (Fig. 5b). Moreover, the ECAR by macrophages after the addition of glucose (i.e., due to glycolysis) decreased significantly in FoxO1-deficient M0 macrophages (Fig. 5c), and the maximum capacity to generate ATP by glycolysis (glycolytic capacity) also decreased (Fig. 5d). Besides, we found that the oxidative phosphorylation level was higher in FoxO1-deficient macrophages than that in normal macrophages, especially the oxygen consumption ratio (Supplementary Fig. S5). In addition, the ATP level of cultured macrophage decreased in FoxO1-deficient macrophages (Fig. 5e, f). The link between the metabolic switch and macrophage function was confirmed by experiments with the glycolysis inhibitor 2-Deoxy-D-glucose (2-DG). After pre-treatment with 2-DG, the proportions of B16 cells co-cultured with FoxO1 ${ }^{\mathrm{mKO}}$ or normal macrophages both increased significantly, which means that the inhibition of glycolysis with 2-DG suppressed the macrophage anti-tumor function including phagocytosis (Fig. 6a, b). In addition, FoxO1-deficient macrophages showed a higher B16 cell count than control macrophages, regardless of 2DG treatment or not (Fig. 6a, b). Although, the inhibited glycolysis activity with 2-DG increased the co-cultured tumor cell counts, which is similar with FoxO1 deficiency related lower glycolysis level. The FoxO1-deficient macrophages still benefited the co-cultured B16 cells compared with 2-DG pre-treated control macrophages, considering the higher OXPHOS activity of FoxO1-deficient macrophages (Fig. 6a, b). Furthermore, the same results were obtained for FoxO1-small interfering RNA-KO macrophages and control macrophages co-cultured with B16F10 ${ }^{\text {Cherry }}$ cells (Fig. 6c).

\section{Discussion}

Our previous research found that FoxO1 acts as a regulator of anti-tumor effect in TAMs, and we found a connection between hypoxia, FoxO1, MHC-II, and tumor growth. These results suggested that lower expression of FoxO1 might be the cause of the pro-tumor M2-like phenotypes of TAMs, but the underlying mechanisms remained unclear ${ }^{37,38,40,41}$. Here we demonstrate that FoxO1 deficiency causes M0 macrophages to resemble alternatively activated (M2) macrophages through downregulating glycolytic function. We first found a similarity between FoxO1 ${ }^{\mathrm{mKO}}$ M0 macrophages and M2 macrophages at the transcriptome level. As mRNA expression does not always match with protein levels due to the translation process and post-translation modifications ${ }^{42}$, we performed a proteomics analysis to validate our transcriptome results (Fig. 1). The M2-like features of FoxOl ${ }^{\mathrm{mKO}}$ macrophages, including the inflammatory response, phagocytic activity, and lymphocyte migration, were confirmed by GSEA and quantitative real-time PCR (Fig. 2 and Supplementary Fig. S1). Next, we analyzed migration and phagocytosis using an HCS system. We observed limited migration of $F o x O 1^{\mathrm{mKO}}$ macrophages compared with control macrophages. In addition, phagocytosis was examined in a co-culture system with B16F10 ${ }^{\text {mCherry }}$ cells. Although the phagocytosis process could be captured in time-lapse movies (Supplementary Fig. S3C and Supplementary Video S2) and by immunofluorescence results (Supplementary Fig. S3B and Supplementary Video 


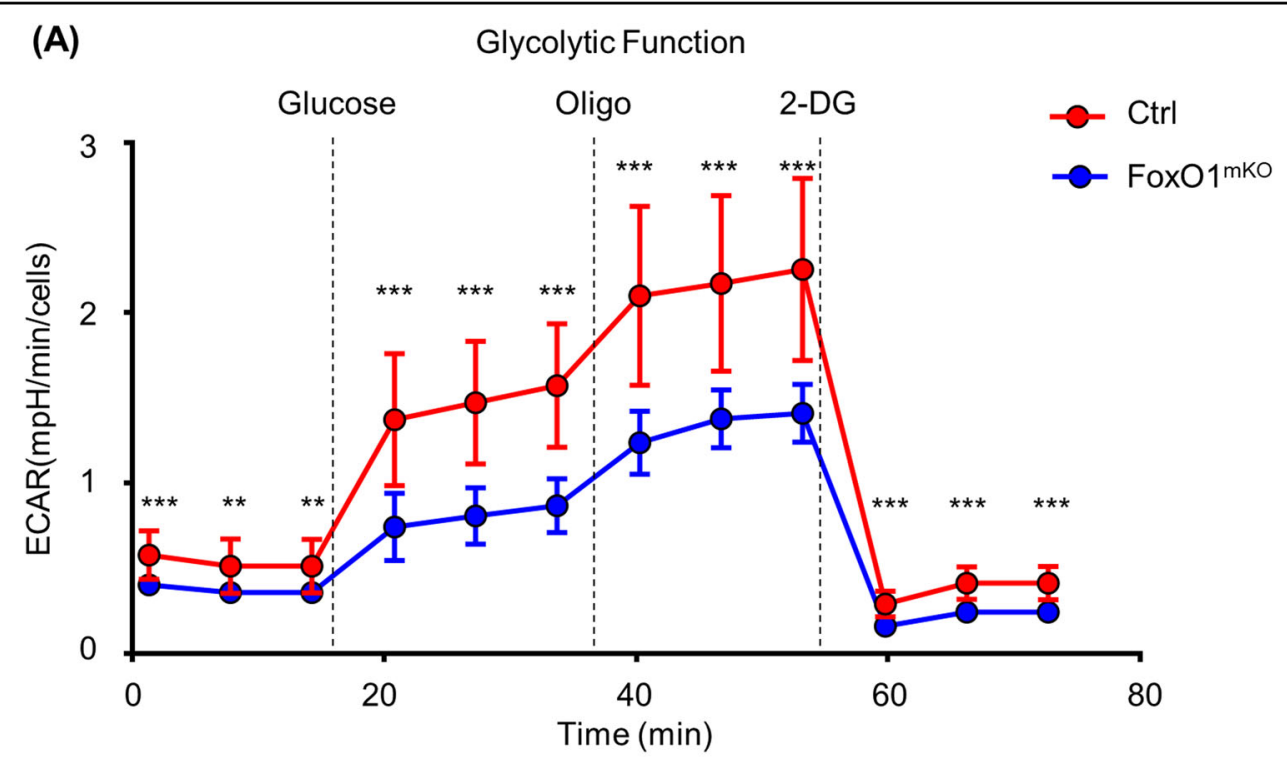

(B)

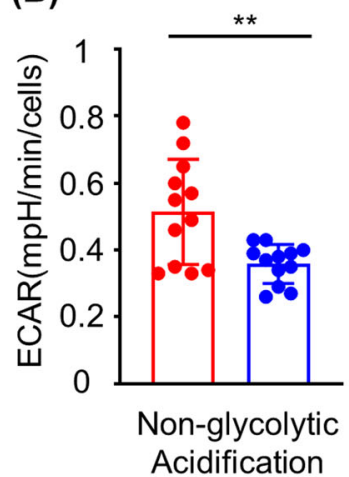

(E)
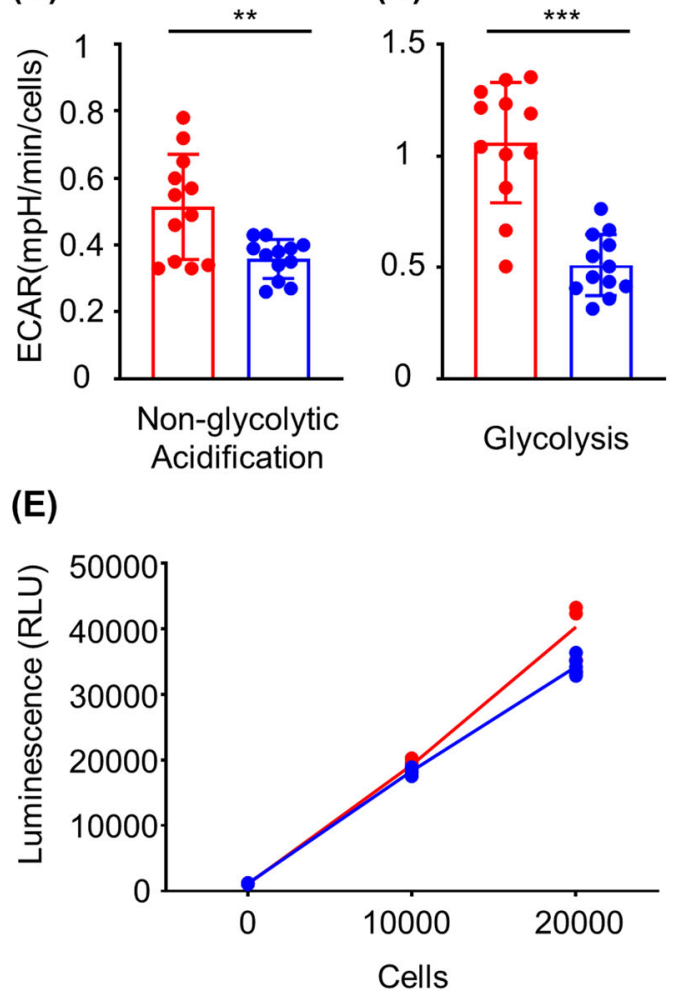

(D)

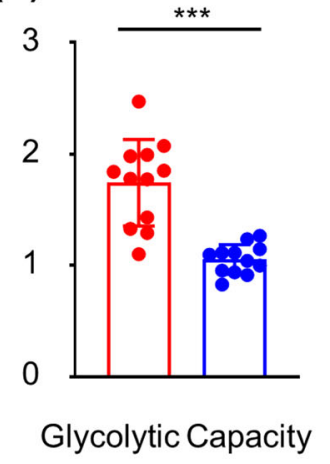

(F)

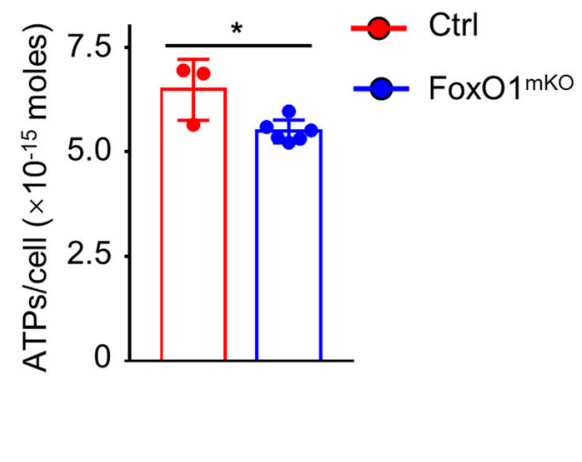

Fig. 5 Glycolysis stress test showed a decrease in glycolytic activity in FoxO1 conditional knockout macrophages. a Kinetic ECAR response of FoxO $1^{\mathrm{mKO}}$ (blue line) and control (red line) macrophages to glucose $(10 \mathrm{mM})$, oligomycin $(1.0 \mu \mathrm{M})$, and 2-DG $(50 \mathrm{mM})(n \geq 3)$. Macrophages were plated at 80,000 cells per well in XFe96 cell culture plates 1 day prior to the assays. b-d Statistical analysis of (b) non-glycolytic acidification, (c) glycolysis, and (d) glycolytic capacity in FoxO1 conditional knockout macrophages vs. control macrophages. e Luminescent signal of FoxOr ${ }^{\mathrm{mKO}}$ (blue), control (red) macrophages and cell counts. f Statistical analysis of ATP per cells of FoxOlmKO (blue), control (red) macrophages $(n \geq 3)$. ${ }^{*} P<0.05$; **P $P$ $0.01 ;{ }^{* *} P<0.001$.

S3A, B), it remained hard to quantify the phagocytosis rate. Hence, we used the relative B16 counts to evaluate the relative phagocytic activity, and we found that $F o x O 1^{\mathrm{mKO}}$ macrophages showed a weaker tumor elimination function (Fig. 3 and Supplementary Video S1A, B). As the metabolic switch functionally shaped the macrophages ${ }^{11,19}$, we next 

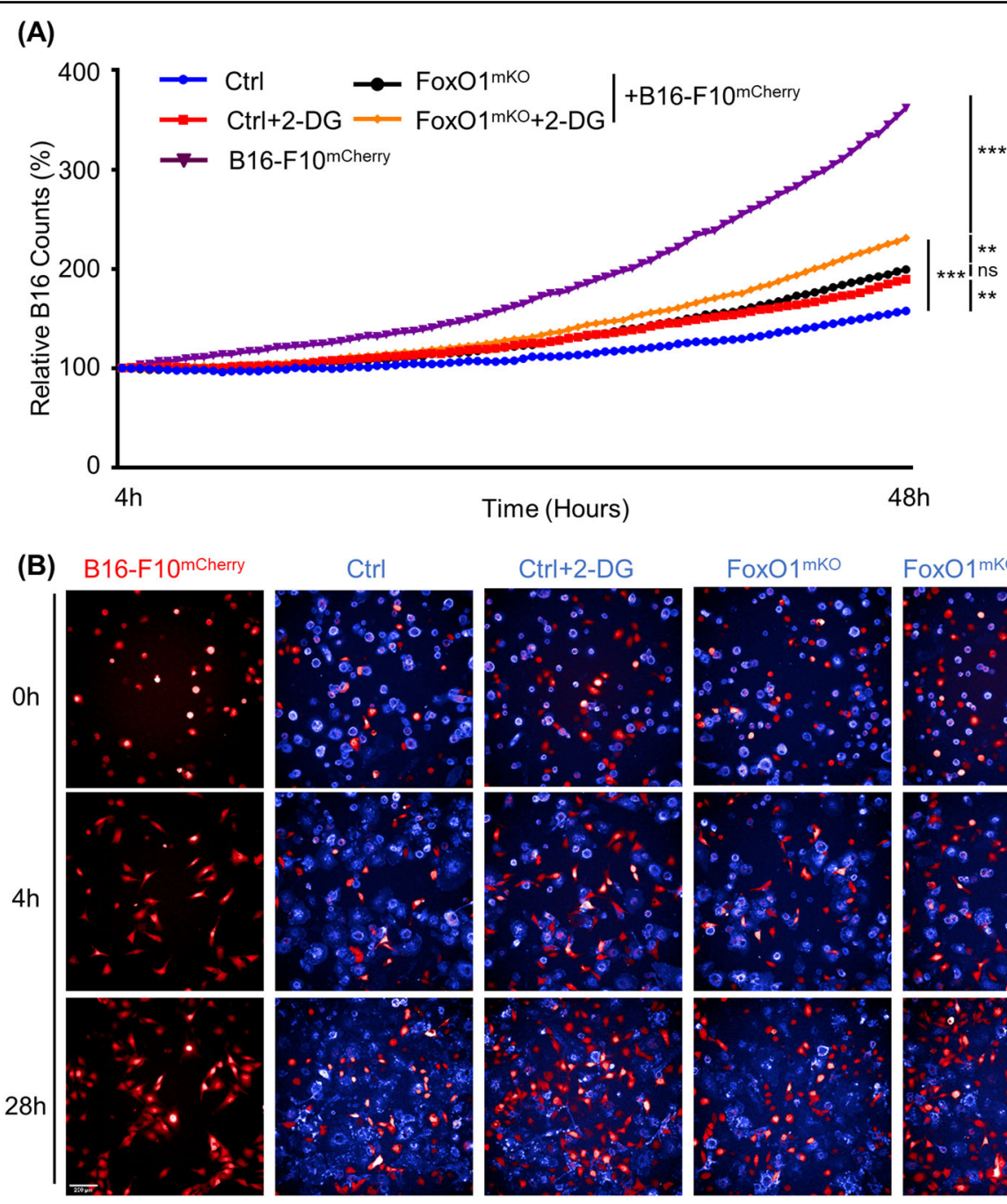

(C)

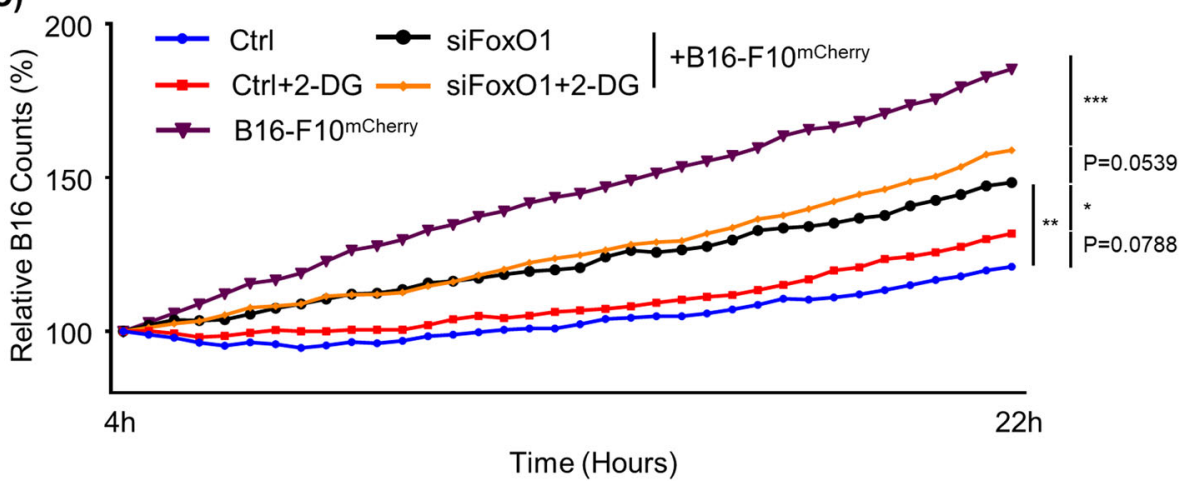

Fig. 6 Glycolysis inhibitor 2-DG exacerbated FoxO1-deficient macrophages induced higher tumor count. a Dynamic B16-F10 mCherry counts of co-cultured B16 cells with FoxO1 ${ }^{\mathrm{mKO}}$ (black), control (blue), and FoxO1 ${ }^{\mathrm{mKO}}$ and control pre-treated with $10 \mathrm{mM}$ 2-DG for $1 \mathrm{~h}$ (orange and red, respectively) relative to the cell count after $4 \mathrm{~h}(n \geq 9)$. $\mathbf{b} \mathrm{HCS}$ images showing the co-culture results of (right) FoxO $1^{\text {mKO }}$ macrophages pre-treated with 2-DG and B16-F10 mCherry cells (second from the right) FoxO $1^{\text {mKo }}$ macrophages and B16-F10 ${ }^{\text {mCherry }}$ cells, (middle) macrophages pre-treated with 2-DG and B16-F10 mCherry cells, (second from the left) macrophages and B16-F10 mCherry cells, and (left) B16-F10 ${ }^{\text {mCherry }}$ cells only, at 0, 4, and 28 h. Blue cells are macrophages and red cells are B16-F10 cells. Scale bar, $200 \mu \mathrm{m}$. c Dynamic B16-F10 ${ }^{\text {mCherry }}$ counts of co-cultured B16 cells with siRNAknockout FoxO1 macrophages (black), control macrophages (blue), and siRNA-knockout FoxO1 macrophages and control macrophages pre-treated with $10 \mathrm{mM} 2-\mathrm{DG}$ for $1 \mathrm{~h}$ (orange and red, respectively) relative to the cell count after $4 \mathrm{~h}(n \geq 9)$. ${ }^{*} P<0.05 ;{ }^{* *} P<0.01 ;{ }^{* * *} P<0.001$. 
analyzed the GSEA data and found that the glycolytic level was decreased in $F o x O 1^{\mathrm{mKO}}$ macrophages. Combined with our FoxO1 knockdown results in vitro and in vivo, we verified that FoxO1 promotes glycolysis in macrophages at transcription, mRNA and protein levels (Figs. 4 and 5, and Supplementary Figs. S4 and S5). Moreover, decreased glycolytic activity of M0 macrophages increased the B16 counts in the co-culture system (Fig. 6).

It has been reported that the metabolic state determines macrophage function, especially glycolysis and oxidative phosphorylation ${ }^{11,19}$. In addition, FoxO1 was found to be an essential regulator of vascular growth that couples metabolic and proliferative activities in endothelial cells ${ }^{27}$. However, little was known about the regulatory effects of FoxO1 on the metabolic state of macrophages. Here we discovered that FoxO1 deficiency significantly decreases the glycolytic activity of macrophages. Using the glycolysis inhibitor 2-DG, we proved that decreased glycolytic activity leads to an increased B16 count in the co-culture system. These results are consistent with previous data of FoxO1-KO macrophages, in which FoxO1 deficiency and relative B16 counts were compared with those of normal resting macrophages (Fig. 6). In summary, our study shows that FoxO1 regulates anti-tumor effect (including phagocytosis) of macrophages through changing the metabolic hemostasis. This may explain why the upregulation of FoxO1 is beneficial in several types of can$\mathrm{cer}^{31-33}$, i.e., FoxO1 deficiency drives resting macrophages to an M2-like/TAM-like phenotype and promotes melanoma growth, as proved by our previous work ${ }^{38}$.

Combining the powerful in vitro culture system and multi-omics analysis, we have drawn a connection between FoxO1, glycolysis, macrophages and phagocytosis of tumor cells. However, several questions remain. For example, macrophage spontaneous moving speed was measured at three frames per hour, but they moved in random directions, so the speeds we calculated might not reflect their true average speed. Under physiological and pathological conditions, macrophages changed and reached their proper place in a specific environment to perform their function through random migration and directionally migration ${ }^{43,44}$. Macrophage mobility to scavenge nearby bacteria and dead cells is required for effective phagocytosis ${ }^{45}$. Thus, we showed macrophage mobility to partially represent their ability of phagocytosis. However, the relation between FoxO1, phagocytosis, random migration, and the chemotactic ability of macrophages remains to be further explored. Then, whether the actual functional readout of FoxO1 on macrophages function is phagocytosis remains unclear. It's better to design another definitive and quantitative experiment defining phagocytosis besides the HCS co-culture system of macrophages and B16-F10 ${ }^{\text {mCherry }}$ cells (Supplementary Fig. S3B, C and Supplementary Videos S2 and S3A, B). Moreover, although we proved reduced glycolysis level and up oxidative phosphorylation level in FoxO1-deficient macrophages (Figs. 4 and 5, and Supplementary Figs. S4 and S5), we need more further study on the in vivo effects of FoxOl on the metabolic switch and the direct consequences for tumor growth. This could be examined with a TAM-specific glycolysis inhibitor or enhancer paired with FoxO1 deficiency. In our previous work, we showed that tumor growth accelerated in myeloid FoxO1 conditional $\mathrm{KO}$ mice ${ }^{38}$. Although TAMs exhibited reduced FoxO1 expression in many tumors, such as mice inoculated with MC38, Hep1-6, or LLC cells (GEO: GSE76033) and human endometrial cancer (GEO: GSE117970), the expression of FoxO1 in TAMs population remain complicated and heterozygous (Supplementary Fig. S6) ${ }^{46,47}$. These all open up ideas for targeting the metabolism of specific subsets of TAMs through interfering FoxO1 as potential clinical treatment, such as nanomaterials. Then, the missing of M1 and M2 macrophages in some experiments design led to multiple misunderstanding, it's better to use those macrophages as control cells in all experiment besides what we had done (Figs. 1 and 3, and Supplementary Fig. S4). Finally, the specific mechanisms by which $F o x O 1$ deficiency decreases the expression of glycolytic enzymes remain unclear. The fact that we found that the expression of several Wnt genes (Wnt9a, Wnt7a, and Wnt5b) was increased upon FoxO1 $\mathrm{KO}$, and the observation that Wnt plays an important role in glycolysis ${ }^{48}$ suggests that Wnt signaling is involved, but this hypothesis requires further study. However, STAT6 and STAT3 genes showed no difference in our transcriptomics data. Besides, the correlation of FoxO1 and STAT6 expression in clustered macrophages from singlecell RNA sequence database of hepatocellular carcinoma and adjacent tissue ${ }^{49}$ showed no difference (Supplementary Fig. S7A). Then, C/EBP $\beta$ mRNA level decreased in FoxO1deficient macrophages tested by real-time PCR (Supplementary Fig. S7B). $C / E B P \beta$ is thought as an positive regulator of glycolysis inducing cancer-type metabolic reprogramming ${ }^{50,51}$. Overall, all the above investigations need to be further proved in future research.

According to our experimental results, glycolysis level decreases (Fig. 5), oxidative phosphorylation level increases (Supplementary Fig. S5) and ATP production level (Fig. 5) decreases in FoxO1-deficient macrophages. It's known that the ATP-producing ability of oxidative phosphorylation is much higher than that of glycolysis, the result of ATP reduction is worthy of discussion and analysis. Studies have shown that compared with resting macrophages, the intracellular ATP content of M2 macrophages under $I L-4$ polarization is unchanged, but the ratio of AMP/ATP increases and the ratio of ATP/ADP decreases significantly ${ }^{10}$. However, the extracellular ATP content of M1 macrophages under LPS-induced polarization is increased ${ }^{52}$. We can conclude that the actual 
ATP level of macrophages have nothing to do with the main metabolic pathway. That is, the ATP content of macrophages dominated by glycolysis (relatively low ATP production) is not definitely lower than that dominated by oxidative phosphorylation (relatively high ATP production), because different cells consume different energy in catabolic processes such as signal pathway activation and cytokine secretion. For example, under the condition of $I L-4$ stimulation, the macrophage JAK-STAT6 signaling pathway is activated and the g-phosphate residues of ATP is transferred to STAT6 for phosphorylation, thereby regulating the downstream signaling pathway ${ }^{53}$.

\section{Materials and methods \\ Mice}

C57BL/6 wild-type mice were purchased from Hunan SLAC Laboratory Animal Co., Ltd (China). Lys ${ }^{\mathrm{Cre} /+}$ mice $\left(\mathrm{B} 6.129 \mathrm{P} 2-L y z 2^{\operatorname{tm1} 1(\mathrm{cre}) I f o} / \mathrm{J}\right)$ were kindly provided by Dr. Bin Gao from NIH, Bethesda, MD. In addition, LoxPflanked FoxO1 (FoxO1 $\left.{ }^{\mathrm{fl} / \mathrm{fl}}\right)$ mice were provided by Dr. Qinghua Shi from University of Science and Technology of China (Hefei, Anhui, China). Lys ${ }^{\mathrm{Cre} /+} \mathrm{FoxO}^{\mathrm{f} / \mathrm{fl}}$ (FoxO1 $1^{\mathrm{mKO}}$ or $\mathrm{KO}$ for short) and its littermates $\mathrm{Lys}^{+/+}$ FoxOl ${ }^{\mathrm{fl} / \mathrm{fl}}$ (control or ctrl) mice used in this study have been previously described ${ }^{37,38}$. Health experimental mice and littermate mice were randomly selected from the mixed feeding cage and used for subsequent research at age of 8-12 weeks. All mice used in this study were bred under specific-pathogen-free conditions according to the guidelines for the Care and Use of Laboratory Animals of the South China University of Technology (Guangzhou, Guangdong, China).

\section{Cell lines}

B16-F10 ${ }^{\text {mCherry }}$ cell line were kindly provided by Dr. Jun Wang from South China University of Technology. Briefly, B16-F10 cells were infected with mCherry fluorescence expression plasmid packaged lentiviruses and B16-F10 ${ }^{\text {mCherry }}$ cells were selected with puromycin ${ }^{54}$.

\section{Bone marrow-derived macrophages}

Bone marrow cells were isolated with phosphatebuffered saline (PBS) and red blood cell lysis buffer (Beyotime, China) following a standardized protocol in sterile conditions. In brief, femurs and tibias of hind legs were cut off from killed mice, flushed with PBS, and filtered with 200-mesh filter to get bone marrow cells. Then, cells were centrifuged at $450 \times g$ for $5 \mathrm{~min}$, resuspended with $2 \mathrm{~mL}$ red blood cell lysis buffer, lysed at $4{ }^{\circ} \mathrm{C}$ for $10 \mathrm{~min}$, washed, and resuspended with PBS to eliminate red blood cells. Every $2 \times 10^{7}$ isolated cells were seeded in a $10 \mathrm{~cm}$ dish and cultured with L929-conditioned medium (Dulbecco's modified Eagle medium-high glucose culture medium containing $10 \%$ fetal bovine serum, $100 \mathrm{U}$ Penicillin-Streptomycin, and 15\% L929 supernatant) for $\sim 7$ days, changing culture medium every 2-3 days if necessary. The matured macrophages (M0 macrophage, M0) were digested with $5 \mathrm{mM}$ EDTA at $37^{\circ} \mathrm{C}$ for $5 \mathrm{~min}$ and then seeded on six-well plates in $1 \times 10^{6} / \mathrm{ml}$ cell density for stimulation. LPS (100 ng/ml; Peprotech, USA)

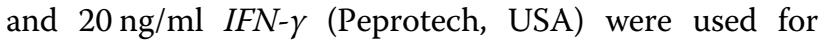
classic activation macrophage (M1 polarization, M1), whereas $10 \mathrm{ng} / \mathrm{ml} \mathrm{IL-4}$ (Peprotech, USA) and $10 \mathrm{ng} / \mathrm{ml}$ IL-13 (Peprotech, USA) were used for alterative activated macrophage (M2 polarization, M2). The treatment of M0 to polarize towards M1 and M2 with cytokines lasted $24 \mathrm{~h}$. A mixture of BMDMs from two FoxO1 ${ }^{\mathrm{mKO}}$ mice or two littermate mice were used as a sample pool for transcriptomics and proteomics analysis. After the stimulation of BMDMs, RNA was extracted with Trizol reagent (Takara, Japan) and used for microarray analysis with the $4 \times 44 \mathrm{~K}$ Agilent Whole Mouse Genome Oligo Microarray (SBC, China). Data were obtained through the RPKM (reads per kilobase of transcript, per million mapped read) matching method and collated as $\log _{2}$ values. PCA, heat map, GSEA, and Pearson's correlation analysis were followed up and analyzed based on the expression of target genes with R. Our transcriptome data can be found on the GEO database (http://www.ncbi.nlm.nih.gov/gds) under the accession number GSE97260.

\section{Proteomics sample preparation}

Cells were gently pelleted with RIPA lysis buffer (Sangon, China) and lysed for $30 \mathrm{~min}$ on ice. Lysed cells were centrifuged at $12,000 \times g$ for $15 \mathrm{~min}$ at $4^{\circ} \mathrm{C}$. The supernatant protein was gently sucked out and quantified with BCA Protein Assay Kit (Thermo, USA). Each aliquot of $50-200 \mu \mathrm{g}$ of proteins was diluted to $300 \mu \mathrm{l}$ with UA buffer $(8 \mathrm{~mol} / \mathrm{L}$ urea in $0.1 \mathrm{~mol} / \mathrm{L}$ Tris- $\mathrm{HCl}, \mathrm{pH} 8.5)$. The sample solution was centrifuged on a $10 \mathrm{k}$ MWCO Concentrator at $10,000 \times g$ for $30 \mathrm{~min}$ at room temperature, twice. Add $100 \mu \mathrm{l}$ iodoacetamide (IAA) solution (UA buffer with $50 \mathrm{mM}$ IAA) to the sample solution and allow the resultant solution to stand for $30 \mathrm{~min}$ at room temperature in the dark for the alkylation reaction. The solution was removed by centrifugation, then washed twice with $200 \mu \mathrm{l}$ UA buffer, and wash twice with $300 \mu \mathrm{l}$ $50 \mathrm{mM} \mathrm{NH}_{4} \mathrm{HCO}_{3}$ buffer at $10,000 \times g$ for $30 \mathrm{~min}$ at $4{ }^{\circ} \mathrm{C}$.

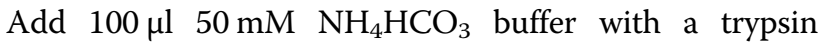
(Thermo, USA)/total protein ratio of $\sim 1: 50$ and incubated at $37^{\circ} \mathrm{C}$ overnight for digestion. Then the flowthrough fractions by centrifugation at $10,000 \times g$ for $15 \mathrm{~min}$ were collected and washed with $50 \mu \mathrm{l}$ of $50 \mathrm{mM}$ $\mathrm{NH}_{4} \mathrm{HCO}_{3}$ buffer twice. Add $10 \%$ trifluoroacetic acid (TFA) for a final TFA concentration of $0.4 \%$ to stop the digestion. The peptide mixture was captured, concentrated, desalted, and eluted with C18 tips (Thermo, 
USA), dried with centrifugal-evaporation-concentrator, and stored at $-80^{\circ} \mathrm{C}$.

\section{Liquid chromatography-mass spectrometry analysis}

The samples were analyzed using a Q Exactive plus mass spectrometers (Thermo, USA) coupled with an EASY-nLC 1200 HPLC system (Thermo, USA) via a nano-electrospray ion source in data-dependent mode same with previous reports $^{55,56}$. In brief, the concentrated peptide mixture resolved in buffer A (0.1\% formic acid (FA)) were loaded onto a $2 \mathrm{~cm}$ self-packed trap column $(100 \mu \mathrm{m}$ inner diameter, packed in-house with nanoViper $\mathrm{C} 18,5 \mu \mathrm{m}$ ) using buffer $\mathrm{A}$ and separated on a $15 \mathrm{~cm}$ liquid chromatography column $(50 \mu \mathrm{m}$ inner diameter, nanoViper $\mathrm{C} 18,2 \mu \mathrm{m})$ over a $75 \mathrm{~min}$ gradient (buffer A, $0.1 \%$ FA in water; buffer B, $20 \%$ buffer A in Acetonitrile) at a flow rate of $300 \mathrm{nl} / \mathrm{min}(0-2 \mathrm{~min}, 2-8 \% \mathrm{~B}$; 2-47 min, 8-28\% B; 47-57 min, 28-44\% B; 57-65 min, $44-100 \%$ B; and $65-75 \mathrm{~min}, 100 \% \mathrm{~B}$ ). The survey scans were operating with a resolution of 70,000 at $350-1800 \mathrm{~m} / \mathrm{z}$ and a maximum injection time of $20 \mathrm{~ms}$. Only two to six charge spectra were fragmented with $27 \%$ normalized, high-energy collision dissociation in the positive-ion mode. Spectra in the ion trap with an AGC target of 5e5 were acquired for $\mathrm{MS}^{2}$ mode and the maximum injection time was $45 \mathrm{~ms}$.

\section{Proteomics identification by Sequest-HT-based database searching}

The mass spectrometry (MS) raw data were searched against the mouse UniProt database (version Jul-03, 2019, 17,019 sequences) using Proteomics Discovery Software (version 2.1, Thermo Fisher Scientific). In the Sequest-HT setting part, we selected trypsin as the proteolytic enzyme and peptides length between 6-144, with 2 missing cleavages sites allowed. The first search mass tolerance and the fragment mass tolerance were 10 p.p.m. and $0.02 \mathrm{Da}$, respectively. Hereafter, the peptide-spectrum-matches and proteins false discovery rates were set to $<1 \%$ released and $0.5 \%$ strictly.

\section{Quality control of the MS platform}

To confirm the performance of MS, we used the Hela cell lysed peptide mixture (lysed and digested with the same protocol with BMDM samples) as the quality-control standard in each Xcalibur sequence before samples.

\section{RNA preparation, reverse transcription, and quantitative real-time PCR}

BMDMs of FoxO1 ${ }^{\mathrm{mKO}}$ and control mice were lysed and stored with Trizol reagent (Invitrogen, USA) for RNA isolation and subsequent RNA sequence. PrimeScript ${ }^{\text {RT }}$ Reagent Kits with gDNA Eraser (Takara, Japan) were used for reverse transcription and quantitative real-time PCR according to the manufacturer's instructions. Results for all target genes were normalized to that of the housekeeping gene $\beta$-Actin was used for calculating $\Delta \Delta \mathrm{Ct}$.
Samples were excluded from the analysis if $\mathrm{Ct}$ value of target genes was out of range. The $5^{\prime}$ to $3^{\prime}$ aligned murine PCR primers (Tsingke, China) used were as follows: $\beta$-Actin, $5^{\prime}$-TTGCTGACAGGATGCAGAAG- ${ }^{\prime}$ and $5^{\prime}$-A CATCTGCTGGAAGGTGGAC-3'; FoxO1, 5' -TGTCAGG CTAAGAGTTAGTGAGCA- $3^{\prime}$ and $5^{\prime}$-GGGTGAAGGGC ATCTTTG-3'; LDHA, 5'-TGTCTCCAGCAAAGACTA CTGT-3' and 5'-GACTGTACTTGACAATGTTGGG A-3'; PKM2, 5'-GCCGCCTGGACATTGACTC-3' and 5'-CCATGAGAGAAATTCAGCCGAG-3'; Eno1, 5'-TGC GTCCACTGGCATCTAC- $3^{\prime}$ and $5^{\prime}$-CAGAGCAGGCGC AATAGTTTTA-3'; Glut1, 5'-CAGTTCGGCTATAACA CTGGTG-3' and 5'-GCCCCCGACAGAGAAGATG-3'; $H K 2,5^{\prime}$-TGATCGCCTGCTTATTCACGG-3' and $5^{\prime}$-AA CCGCCTAGAAATCTCCAGA-3'; IL-4, 5'-GGTCTCA ACCCCCAGCTAGT- $3^{\prime}$ and $5^{\prime}$-GCCGATGATCTCTC TCAAGTGAT-3'.

\section{HCS for macrophage tumor-killing function}

HCS imaging system (PerkinElmer, USA) was used to analyze the tumor-killing function of $\mathrm{KO}$ and control macrophage in confocal mode ${ }^{57,58}$. Every $1 \times 10^{6}$ resuspended polarized macrophages were stained with $2 \mu \mathrm{l}$ Allophycocyanin (APC)-F4/80 antibodies (Biolegend, USA; catalog number 123116) for $15 \mathrm{~min}$, at $4{ }^{\circ} \mathrm{C}$ after being digested with $5 \mathrm{mM}$ EDTA, $5 \mathrm{~min}$, at $37^{\circ} \mathrm{C}$. Spare antibodies with $1 \times$ PBS buffer (PBS), centrifuged at $3000 \times g, 2 \mathrm{~min}$, were washed. Macrophages on the PerkinElmer 96-well plates with $1 \times 10^{4}$ cells per well in $100 \mu \mathrm{l}$ culture medium were counted and seeded. Then, $2 \times 10^{4}$ B16-F10-mCherry tumor cells in $100 \mu \mathrm{l}$ medium were added to the overnight adhered macrophages. The lid was opened and the plate was loaded on the HCS machine immediately, then the image acquisition mode in Harmony software was set. Water objective lens $(20 \times)$ and R-phycoerythrin (PE) (for cancer cells)/APC (for macrophages)/bright-filed channels were used for confocal imaging mode including $200 \mathrm{~ms}$ exposure time, 100\% excitation power, and $1.6 \mu \mathrm{m}$ focus height. Thirteen areas were imaged for each well, with $30 \mathrm{~min}$ fixed interval and 96 time points in $37^{\circ} \mathrm{C}, 5 \% \mathrm{CO}_{2}$. Cell counts and the tumor cell-killing ability were analyzed with Harmony software according to the cell-tracing analysis model. Displayed view fields were randomly selected from the imaging areas of per well, with $8 \mathrm{~h}$ interval after the $8^{\text {th }}$ time point, and the timeline video was composed of representative scene of the wells at 96 time points. All experiments repeated at least twice.

\section{FoxO1-KO virus construction and macrophages infection}

FoxO1-shRNA plasmids (Sigma, US) were kindly provided by Prof. Ping Gao from SCUT. The used FoxO1-shRNA sequence was 5'-CCGGCGGAGGATTG AACCAGTATAACTCGA 
GTTATACTGGTTCAATCCTCCGTTTTTG-3' and 5'CCGGCCGCCAAACACCAGTCTAAATCTCGAGATT TAGACTGGTGTTTGGCGGTTTTTG-3'. FoxO1-shRNA and NTC (negative control shRNA) plasmids paired with $\mathrm{CMV}^{\triangle \mathrm{R} 8.91}$ (expressing 3 required HIV proteins) and PMD.G (expressing the VSV-G envelop proteins) plasmids were packaged for lentivirus with PEI (polyethyleneimine) in 293 T cells. PEI was moved and replaced with normal culture medium $6 \mathrm{~h}$ later. Then, lentivirus was isolated from supernatants and cells after 48 and $72 \mathrm{~h}$ by $3000 \times g 5 \mathrm{~min}$ centrifugation and filtration with $0.45 \mu \mathrm{m}$ filter. Six-well plates cultured BMDMs were infected with $1 \mathrm{ml}$ FoxO1shRNA, and NTC lentivirus and $6 \mu$ l Turbofect infection reagent (Thermo, US) at the second day to sustain for $24 \mathrm{~h}$, or infected cultured BMDMs with Ad5-CMV-Cre-GFP (Vigenebio, China) or control adeno-virus at 400 multiplicity of infection and sustain overnight. Then the macrophages were cultured in complete culture medium with 15\% L929 culture supernatants for another 3 days.

\section{Glycolysis stress test by seahorse}

On the day before acute extracellular flux (XF) assay, seeding $2.0 \times 10^{5}$ cells in growth medium and hydrate sensor cartridge with $200 \mu \mathrm{l}$ water to incubate overnight in $37^{\circ} \mathrm{C}$ without $\mathrm{CO}_{2}$. Also, we changed $180 \mu \mathrm{l} /$ well culture medium to $37^{\circ} \mathrm{C}$ pre-warmed bicarbonate-free low-buffered assay medium ( $\mathrm{pH}$ 7.4) $1 \mathrm{~h}$ prior to the XF assay, prepared the compounds and loaded into the hydrated cartridge ports. For Glycolysis Stress Test, final concentrations were $10 \mathrm{mM}$ glucose in port $\mathrm{A}, 2 \mu \mathrm{M}$ oligomycin in port $\mathrm{B}$, and $50 \mathrm{mM}$ 2-DG in port $\mathrm{C}$. We created and loaded the assay template on the Seahorse (Agilent, USA), calibrated the sensors with XF calibrant (pH 7.4), and ran the XF assay ${ }^{39,59}$.

\section{2-NBDG glucose uptake assay and ATP production assay}

2-NBDG (2-(N-(7-Nitrobenz-2-oxa-1,3-diazol-4-yl)Amino)2-Deoxyglucose), a fluorescent glucose analog, was used to evaluate cell glucose uptake capacity (BioVision, USA). BMDMs were seeded on the 24-well tissue culture plate in $4 \times 10^{4} /$ well cell density to tested the glucose uptake level according to the standard operation procedure. The amount of ATP present of viable cells in culture were quantified by CellTiter-Glo® 2.0 Assay (Promega, USA). BMDMs were seeded on the 96-well tissue culture plate in $2 \times 10^{4} /$ well or $1 \times 10^{4} /$ well cell density to tested the ATP production level according to the standard protocol. ATP solutions $(1 \mu \mathrm{M}$ to $10 \mathrm{nM}$ ) were used to generate the standard curve.

\section{Statistical analysis}

All statistical data were presented as mean \pm SD. For unpaired samples $(n \geq 3)$, normality test, and homogeneity of variance test were conducted (GraphPad Prism, Version 7.0). If normality and variance homogeneity are met, use twotailed unpaired Student's $t$-test; if not, use Mann-Whitney test. For multiple sets of samples, if they meet the normal distribution, use one-way analysis of variance test, otherwise use non-parametric test. $P$-value $<0.05$ was considered as statistically significant $\left({ }^{*} P<0.05 ;{ }^{* *} P<0.01\right.$; $\left.{ }^{* * *} P<0.001\right)$.

\section{Author details}

'Liver Immunology Laboratory, School of Life Sciences, University of Science and Technology of China, Hefei 230022 Anhui, China. ${ }^{2}$ Chronic Disease Laboratory, Institutes for Life Sciences and School of Medicine, South China University of Technology, Guangzhou 510006 Guangzhou, China. ${ }^{3}$ Department of Rheumatology and Immunology, The Third Affiliated Hospital of Southern Medical University, Guangzhou 510630 Guangdong, China

\section{Funding}

This work is supported by the Program for Guangdong Introducing Innovative and Entrepreneurial Teams (2017ZT075054), the National Natural Science Foundation of China (81901652, 81801607, and 81901653), China Postdoctoral Science Foundation (2019M650201), and State Key Laboratory of Pathogenesis, Prevention, Treatment of Central Asian High Incidence Diseases Fund, China (SKL-HIDCA-2018-4).

\section{Code availability}

All transcriptomics data were analyzed with $\mathrm{R}$ (Version 3.6.3, on the Rstudio platform). After extracting the original data with Proteomics Discovery software, the subsequent graph analysis of the proteome was also completed on R. All code that support the findings of this study are available from the corresponding author upon reasonable request.

\section{Ethics statement}

This study was carried out in accordance with the recommendations of Guide for the Care and Use of Laboratory Animals, SCUT Animal Care and Use Committee. The protocol was approved by the SCUT Laboratory Animal Research Center.

Conflict of interest

The authors declare that they have no conflict of interest.

\section{Publisher's note}

Springer Nature remains neutral with regard to jurisdictional claims in published maps and institutional affiliations.

Supplementary Information accompanies this paper at (https://doi.org/ 10.1038/s41419-020-02982-0).

Received: 10 January 2020 Accepted: 10 July 2020

Published online: 24 September 2020

\section{References}

1. Raggi, F. et al. Regulation of human macrophage M1-M2 polarization balance by hypoxia and the triggering receptor expressed on myeloid cells-1. Front. Immunol. 8, 1097 (2017).

2. Wynn, T. A., Chawla, A. \& Pollard, J. W. Macrophage biology in development, homeostasis and disease. Nature 496, 445-455 (2013).

3. MacParland, S. A. et al. Single cell RNA sequencing of human liver reveals distinct intrahepatic macrophage populations. Nat. Commun. 9, 4383 (2018).

4. Tsuchiya, K. et al. Homozygosity for an allele encoding deacetylated FoxO1 protects macrophages from cholesterol-induced inflammation without increasing apoptosis. Arterioscler. Thromb. Vasc. Biol. 31, 2920-2928 (2011).

5. Martinez, F. O. et al. Genetic programs expressed in resting and IL-4 alternatively activated mouse and human macrophages: similarities and differences. Blood 121, e57-e69 (2013).

6. Lang, R., Patel, D., Morris, J. J., Rutschman, R. L. \& Murray, P. J. Shaping gene expression in activated and resting primary macrophages by IL-10. J. Immunol. 169, 2253-2263 (2002).

7. Mosser, D. M. \& Edwards, J. P. Exploring the full spectrum of macrophage activation. Nat. Rev. Immunol. 8, 958-969 (2008). 
8. Gordon, S. \& Martinez, F. O. Alternative activation of macrophages: mechanism and functions. Immunity 32, 593-604 (2010).

9. Kelly, B. \& O'Neill, L. A. Metabolic reprogramming in macrophages and dendritic cells in innate immunity. Cell Res. 25, 771-784 (2015).

10. Wang, F. et al. Glycolytic stimulation is not a requirement for M2 macrophage differentiation. Cell Metab. 28, 463-475 (2018). e4.

11. Viola, A., Munari, F., Sanchez-Rodriguez, R., Scolaro, T. \& Castegna, A. The metabolic signature of macrophage responses. Front. Immunol. 10, 1462 (2019).

12. Pathria, P., Louis, T. L. \& Varner, J. A. Targeting tumor-associated macrophages in cancer. Trends Immunol. 40, 310-27. (2019).

13. Komohara, Y. \& Takeya, M. C. A. Fs and TAMs: maestros of the tumour microenvironment. J. Pathol. 241, 313-315 (2017).

14. Murray, P. J. Macrophage polarization. Annu. Rev. Physiol. 79, 541-66. (2017).

15. Sica, A. et al. Macrophage polarization in tumour progression. Semin. Cancer Biol. 18, 349-55. (2008).

16. Vitale, I., Manic, G., Coussens, L. M., Kroemer, G. \& Galluzzi, L. Macrophages and metabolism in the tumor microenvironment. Cell Metab. 30, 36-50 (2019).

17. Md-B, N. et al. Aerobic glycolysis is a metabolic requirement to maintain the M2-like polarization of tumor-associated macrophages. Biochim. Biophys. Acta Mol. Cell Res. 1867, 118604 (2020).

18. Van den Bossche, J. \& van der Windt, G. J. W. Fatty acid oxidation in macrophages and T cells: time for reassessment? Cell Metab. 28, 538-540 (2018).

19. Diskin, C. \& Palsson-McDermott, E. M. Metabolic modulation in macrophage effector function. Front Immunol. 9, 270 (2018).

20. Netea-Maier, R. T., Smit, J. W. A. \& Netea, M. G. Metabolic changes in tumor cells and tumor-associated macrophages: a mutual relationship. Cancer Lett. 413, 102-109 (2018).

21. Mills, E. L. \& O'Neill, L. A. Reprogramming mitochondrial metabolism in macrophages as an anti-inflammatory signal. Eur. J. Immunol. 46, 13-21 (2016).

22. Galvan-Pena, S. \& O'Neill, L. A. Metabolic reprograming in macrophage polarization. Front. Immunol. 5, 420 (2014).

23. Sica, A., Bleve, A. \& Garassino, M. C. Membrane cholesterol regulates macrophage plasticity in cancer. Cell Metab. 29, 1238-40. (2019).

24. Ji, L. et al. Slc6a8-mediated creatine uptake and accumulation reprogram macrophage polarization via regulating cytokine responses. Immunity $\mathbf{5 1}$ 272-284 (2019). e7.

25. Colegio, O. R. et al. Functional polarization of tumour-associated macrophages by tumour-derived lactic acid. Nature 513, 559-563 (2014).

26. Lampropoulou, $\vee$. et al. Itaconate links inhibition of succinate dehydrogenase with macrophage metabolic remodeling and regulation of inflammation. Cell Metab. 24, 158-166 (2016).

27. Wilhelm, K. et al. FOXO1 couples metabolic activity and growth state in the vascular endothelium. Nature 529, 216-220 (2016).

28. Miyauchi, T. et al. Up-regulation of FOXO1 and reduced inflammation by betahydroxybutyric acid are essential diet restriction benefits against liver injury. Proc. Natl Acad. Sci. USA 116, 13533-13542 (2019).

29. Pajvani, U. B. et al. Inhibition of Notch signaling ameliorates insulin resistance in a FoxO1-dependent manner. Nat. Med. 17, 961-967 (2011).

30. Yan, $\mathrm{H}$. et al. Estrogen improves insulin sensitivity and suppresses gluconeogenesis via the transcription factor Foxo1. Diabetes 68, 291-304 (2019).

31. Chen, J., Huang, Q. \& Wang, F. Inhibition of FoxO1 nuclear exclusion prevents metastasis of glioblastoma. Tumour Biol. 35, 7195-200. (2014).

32. Lu, H., Liu, P., Pan, Y. \& Huang, H. Inhibition of cyclin-dependent kinase phosphorylation of FOXO1 and prostate cancer cell growth by a peptide derived from FOXO1. Neoplasia 13, 854-63. (2011).

33. Jiang, G. et al. Isorhapontigenin (ISO) inhibits invasive bladder cancer formation in vivo and human bladder cancer invasion in vitro by targeting STAT1/ FOXO1 axis. Cancer Prev. Res. 9, 567-80. (2016).

34. Chung, $\mathrm{S}$. et al. FoxO1 is a critical regulator of M2-like macrophage activation in allergic asthma. Allergy 74, 535-48. (2019).
35. Fan, W. et al. FoxO1 regulates Tlr4 inflammatory pathway signalling in macrophages. EMBO J. 29, 4223-36. (2010).

36. Chung, S. et al. Distinct role of FoxO1 in M-CSF- and GM-CSF-differentiated macrophages contributes LPS-mediated IL-10: implication in hyperglycemia. J. Leukoc. Biol. 97, 327-39. (2015).

37. Wang, Y. C. et al. Forkhead Box $\mathrm{O} 1$ regulates macrophage polarization following Staphylococcus aureus infection: experimental murine data and review of the literature. Clin. Rev. Allergy Immunol. 51, 353-69. (2016).

38. Yang, J. B. et al. FoxO1 is a regulator of MHC-II expression and antitumor effect of tumor-associated macrophages. Oncogene $\mathbf{3 7}$ 1192-204. (2018)

39. Van den Bossche J., Baardman J., de Winther M. P. Metabolic characterization of polarized $\mathrm{M} 1$ and $\mathrm{M} 2$ bone marrow-derived macrophages using real-time extracellular flux analysis. J. Vis. Exp. 28, 53424 (2015).

40. Huang, L., Xu, H. \& Peng, G. TLR-mediated metabolic reprogramming in the tumor microenvironment: potential novel strategies for cancer immunotherapy. Cell. Mol. Immunol. 15, 428-437 (2018).

41. Zhang W., et al. The metabolic regulator Lamtor5 suppresses inflammatory signaling via regulating mTOR-mediated TLR4 degradation. Cell. Mol. Immunol. https://doi.org/10.1038/s41423-019-0281-6 (2019).

42. Zaher, H. S. \& Green, R. Quality control by the ribosome following peptide bond formation. Nature 457, 161-166 (2009).

43. Petrie, R. J., Doyle, A. D. \& Yamada, K. M. Random versus directionally persistent cell migration. Nat. Rev. Mol. Cell Biol. 10, 538-49. (2009).

44. Andrew, N. \& Insall, R. H. Chemotaxis in shallow gradients is mediated independently of Ptdlns 3-kinase by biased choices between random protrusions. Nat. Cell Biol. 9, 193-200 (2007)

45. De, A. \& Chattopadhyay, S. The variation in cytoplasmic distribution of mouse peritoneal macrophage during phagocytosis modulated by mangiferin, an immunomodulator. Immunobiology 214, 367-76. (2009).

46. Poczobutt, J. M. et al. Expression profiling of macrophages reveals multiple populations with distinct biological roles in an immunocompetent orthotopic model of lung cancer. J. Immunol. 196, 2847-59. (2016).

47. Cassetta, L. et al. Human tumor-associated macrophage and monocyte transcriptional landscapes reveal cancer-specific reprogramming, biomarkers, and therapeutic targets. Cancer Cell. 35, 588-602 (2019). e10.

48. Pate, K. T. et al. Wnt signaling directs a metabolic program of glycolysis and angiogenesis in colon cancer. EMBO J. 33, 1454-1473 (2014).

49. Zhang, Q. et al. Landscape and dynamics of single immune cells in hepatocellular carcinoma. Cell 179, 829-845 (2019). e20.

50. Ackermann, T. et al. C/EBPbeta-LIP induces cancer-type metabolic reprogramming by regulating the let-7/LIN28B circuit in mice. Commun. Bio/ 2, 208 (2019).

51. Li, W. et al. Aerobic glycolysis controls myeloid-derived suppressor cells and tumor immunity via a specific CEBPB isoform in triple-negative breast cancer. Cell Metab. 28, 87-103 (2018). e6.

52. Dosch $M_{\text {., }}$ et al. Connexin-43-dependent ATP release mediates macrophage activation during sepsis. Elife. 8, e42670 (2019).

53. Shuai, K. \& Liu, B. Regulation of JAK-STAT signalling in the immune system. Nat. Rev. Immunol. 3, 900-911 (2003).

54. van der Weyden, L. et al. Genome-wide in vivo screen identifies novel host regulators of metastatic colonization. Nature 541, 233-236 (2017).

55. Eckert, M. A. et al. Proteomics reveals NNMT as a master metabolic regulator of cancer-associated fibroblasts. Nature 569, 723-728 (2019).

56. Jiang, $Y$. et al. Proteomics identifies new therapeutic targets of early-stage hepatocellular carcinoma. Nature 567, 257-261 (2019).

57. Thomas, N. High-content screening: a decade of evolution. J. Biomol. Screen 15, 1-9 (2010).

58. Mattiazzi Usaj, M. et al. High-content screening for quantitative cell biology. Trends Cell Biol. 26, 598-611 (2016).

59. Tanner, L. B. et al. Four key steps control glycolytic flux in mammalian cells. Cell Syst. 7, 49-62 (2018) 